\section{Prevalência e fatores associados à inatividade física nos deslocamentos para escola em adolescentes}

\author{
Prevalence of physical inactivity and associated \\ factors among adolescents commuting to school
}

\author{
1 Programa Associado de \\ Pós-graduação em Educação \\ Física, Universidade de \\ Pernambuco/Universidade \\ Federal da Paraíba, Recife, \\ Brasil. \\ 2 Escola Superior \\ de Educação Física, \\ Universidade de Pernambuco, \\ Recife, Brasil. \\ 3 Centro de Ciências da \\ Saúde, Universidade Federal \\ de Paraíba, João Pessoa, \\ Brasil. \\ Correspondência \\ C. M. Santos \\ Programa Associado de Pós- \\ graduação em Educação \\ Física, Universidade de \\ Pernambuco/Universidade \\ Federal da Paraíba. \\ Rua Arnóbio Marques 310 \\ Campus Universitário, Recife, \\ PE 50100-130, Brasil. \\ carlinhams@gmail.com
}

\begin{abstract}
The aim of this study was to verify the prevalence of physical inactivity and associated factors among adolescents commuting to school. This was an epidemiological study based on secondary data from a sample of 4,207 adolescents (14-19 years). Data were collected through a previously validated questionnaire (GSHS-WHO). Adolescents were classified as "inactive in commuting" when they reported not commuting to school actively (e.g., walking or bicycling) and/or spent less than 20 minutes getting to and from school. 43\% (95\%CI: 41.5-44.5) of adolescents were physically inactive in commuting. Place of residence and maternal schooling were statistically associated with outcome (inactivity in commuting to school) $(p<0.01)$. Among male adolescents, physical inactivity in commuting was significantly associated with age $(p=0.02)$ and skin color $(p=0.04)$. Inactivity in commuting was relatively common when compared to other studies.
\end{abstract}

Motor Activity; Walking; Bicycling; Adolescent
Carla Menêses Santos 1,2

Rildo de Souza Wanderley Júnior 2

Simone Storino Honda Barros 2

José Cazuza de Farias Júnior 3

Mauro Virgilio Gomes de Barros 1,2

Os benefícios para a saúde e o bem-estar decorrentes da prática regular de atividade física estão bem documentados. Estudos epidemiológicos indicam que esta tem um papel fundamental na prevenção de várias doenças $1,2,3,4$ e na melhoria da auto-estima e qualidade de vida 5 . Apesar disso, evidências disponíveis indicam uma tendência de aumento na proporção de indivíduos expostos a baixo nível de atividade física, independentemente da idade 6,7 .

Diante deste cenário, tanto no campo da pesquisa quanto no da intervenção em saúde, vem crescendo o debate sobre as ações que podem ser desenvolvidas a fim de intervir, o mais precocemente possível, para promoção de estilos de vida mais ativos fisicamente ${ }^{8}$. No domínio dos deslocamentos, o incentivo à prática de caminhar e pedalar vem sendo indicado como uma estratégia eficiente e efetiva para aumentar os níveis de atividade física na população jovem 9,10.

O deslocamento fisicamente ativo para escola pode constituir uma importante fonte de atividade física diária 11. Evidências consistentes sugerem que a prática de atividades físicas nos deslocamentos está intimamente associada a diversos desfechos relacionados à saúde, como o aumento dos níveis gerais de atividade física 12 , de aptidão cardiorrespiratória ${ }^{13}$, manutenção da massa corporal 13,14 e redução do risco de acidente vascular cerebral isquêmico ${ }^{3}$. Recentemente, 
um estudo de meta-análise revelou que o deslocamento ativo foi associado com $11 \%$ de redução do risco cardiovascular 15 .

Embora a utilização de modos de transporte fisicamente ativo influenciem positivamente na atividade física habitual dos jovens, a proporção de crianças e adolescentes que caminham ou andam de bicicleta para a escola vem diminuindo 16. No Brasil, ainda há poucos levantamentos abordando a prática de atividade física dos sujeitos em seus deslocamentos 17 . Os estudos que focalizaram esta temática identificaram que $20 \%$ a 30\% dos jovens parecem estar expostos à inatividade física nos deslocamentos 18,19,20.

Pesquisa conduzida no Estado de Santa Catarina demonstrou que $20 \%$ dos estudantes (7-10 anos) usavam um modo de transporte fisicamente inativo para ir à escola 18 . Hallal et al. 19 verificaram que $27,2 \%$ dos adolescentes (10-12 anos) pelotenses utilizavam ônibus, carro ou moto no deslocamento para a escola. No estudo realizado por Silva \& Lopes 20, 30\% dos escolares (7-12 anos) da cidade de João Pessoa foram considerados fisicamente inativos nos deslocamentos.

Avaliar os níveis de atividade física por domínio pode contribuir para identificação dos fatores que determinam as opções de transporte que os jovens utilizam e, consequentemente, orientar melhor o planejamento de intervenções intersetoriais, desenvolvidas como parte dos programas de promoção da atividade física. Nesse sentido, o objetivo do presente estudo foi verificar a prevalência e identificar fatores associados à inatividade física nos deslocamentos para escola em adolescentes.

\section{Métodos}

Estudo transversal baseado na análise secundária de dados de um levantamento epidemiológico de abrangência estadual e base escolar, intitulado Estilos de Vida e Comportamentos de Risco à Saúde em Estudantes do Ensino Médio no Estado de Pernambuco.

Pernambuco está localizado no centro-leste da Região Nordeste do Brasil e tem população de aproximadamente oito milhões de habitantes. A renda per capita anual é de $\mathrm{R} \$ 6.528,0021$, e o estado apresenta o quinto menor índice de desenvolvimento humano da região (IDH $=0,71$, ano-base 2005) (Programa de Desenvolvimento das Nações Unidas. Ranking do IDH dos estados em 2005. http://www.pnud.org.br/pobreza_desigualda $\mathrm{de} /$ reportagens $/$ index.php?id01=3039\&lay=pde, acessado em 26/Set/2009).

O protocolo do estudo foi aprovado pelo Comitê de Ética em Pesquisa com seres humanos do Hospital Agamenon Magalhães em Recife. A participação dos sujeitos foi voluntária e anônima, adotando-se a utilização de termo negativo de consentimento (parental passive consent form). Não foi permitido uso de qualquer tipo de identificação pessoal nos instrumentos a fim de garantir o anonimato das respostas.

A população-alvo deste estudo foi limitada aos estudantes da rede pública estadual de ensino médio do Estado de Pernambuco, com idade entre 14 e 19 anos. Considerando-se todas as dependências administrativas (federal, estadual, municipal e privada), os estudantes matriculados na rede pública estadual representavam, à época, aproximadamente $80 \%$ do total em todo o estado. No cálculo do tamanho da amostra, foram adotados os seguintes critérios: (i) população estimada em 353 mil estudantes; (ii) intervalo de 95\% de confiança (IC95\%); (iii) poder estatístico de 80\%; (iv) erro amostral tolerável de três pontos percentuais; (v) prevalência estimada em 50\%; (vi) efeito de delineamento amostral estabelecido em quatro vezes o tamanho mínimo da amostra. Adotando-se esses critérios, seria necessário alcançar uma amostra com 4.217 sujeitos.

Recorrendo-se a cálculos de poder estatístico a posteriori, verificou-se que esse dimensionamento amostral permitiria efetuar análise de associação entre as variáveis com possibilidade de detectar como estatisticamente significativas razões de odds (OR) de 1,2 ou superiores. Para isso, considerou-se: (i) prevalência do desfecho (inatividade física nos deslocamentos para escola) entre $39 \%$ e $64 \%$ nos expostos e entre $35 \%$ e $60 \%$ nos não expostos; (ii) poder estatístico de $80 \%$; (iii) nível de $95 \%$ de confiança.

Procurou-se garantir que a amostra selecionada representasse a população-alvo, considerando a sua distribuição conforme região geográfica, período de matrícula (diurno e noturno) e porte das escolas (pequenas, com menos de 200 alunos; médias, com 200 a 499 alunos; grandes, com 500 estudantes ou mais). Alunos matriculados no período da manhã e da tarde foram agrupados numa única categoria (estudantes do período diurno). A distribuição regional foi observada pelo número de escolas existentes em cada uma das 17 Gerências Regionais de Ensino da Secretaria de Educação do Estado.

Para seleção da amostra, recorreu-se a um procedimento de amostragem por conglomerados em dois estágios; a "escola" e a "turma" representaram, respectivamente, as unidades amostrais no primeiro e no segundo estágio. Todas as escolas da rede pública estadual que ofereciam ensino médio regular foram consideradas elegíveis para inclusão no estudo. No primeiro estágio, adotou-se como critério de estratificação a 
densidade de escolas em cada microrregião do estado, segundo porte (tamanho) destas; assim, foram sorteadas, proporcionalmente, mais escolas nas microrregiões onde a densidade era também maior. No segundo estágio, considerouse a densidade de turmas nas escolas sorteadas por período de assistência às aulas (diurno e noturno) como critério para sorteio daquelas nas quais os questionários seriam aplicados. Todos os alunos das turmas sorteadas foram convidados a participar do estudo, independentemente da idade deles.

A coleta dos dados foi realizada no período de abril a outubro de 2006. A aplicação dos questionários foi efetuada em sala de aula, sem a presença dos professores, por seis estudantes de pós-graduação (três profissionais de educação física, duas enfermeiras e um médico), que foram previamente capacitados para participação na coleta de dados. Os sujeitos foram continuamente assistidos pelos aplicadores (sempre dois por turma) para que pudessem esclarecer dúvidas e auxiliar no preenchimento das informações. Após a aplicação, os questionários respondidos por estudantes com idade inferior a 14 e superior a 19 anos foram excluídos.

As informações foram autorreferidas, obtidas mediante aplicação do questionário Global School-based Health Survey, proposto pela Organização Mundial da Saúde (OMS) em colaboração com outras instituições internacionais. O instrumento é constituído por dez módulos: (1) informações pessoais; (2) consumo de álcool e drogas; (3) hábitos alimentares; (4) higiene; (5) sentimentos e relacionamentos; (6) atividades físicas; (7) comportamentos na escola; 8) comportamento sexual; (9) tabagismo e (10) violência. Neste estudo, foram analisadas variáveis demográficas, socioeconômicas, relacionadas à escola e comportamentais.

O questionário foi adaptado e previamente testado em uma amostra de 138 estudantes de ensino médio matriculados em escolas da rede municipal na cidade do Recife. Os resultados indicaram que o instrumento tem boa consistência de medidas (reprodutibilidade), validade de conteúdo e de face. Os indicadores de reprodutibilidade apresentaram um coeficiente de correlação intraclasse de moderados a altos na maioria dos itens do questionário, tendo os coeficientes de concordância (índice kappa) variado de 0,52 a 1,00 . Nas questões empregadas para medida da atividade física, os coeficientes foram de 0,64 ou superiores. O tempo para preenchimento do instrumento foi de aproximadamente 40 minutos.

Após o preenchimento do questionário, foi realizada a mensuração da massa corporal e da estatura, com os estudantes descalços, usando roupas leves e sem portar objetos pesados. A massa corporal foi aferida utilizando balanças digitais portáteis, modelo Sport (marca Plenna, São Paulo, Brasil). A estatura foi avaliada por meio de estadiômetros, modelo 206 (marca Plenna, São Paulo, Brasil), seguindo a padronização proposta na literatura especializada 22. Foram realizadas três mensurações para cada aluno, adotando-se a média destas como a medida final. Valendo-se das medidas de massa e estatura corporal, efetuou-se cálculo do índice de massa corporal $(\mathrm{IMC}=$ massa em quilogramas/ estatura em metros²).

Informações relativas à prática de atividades físicas nos deslocamentos de casa para a escola (variável dependente) foram obtidas através de duas questões: "durante os últimos sete dias, em quantos dias você andou a pé ou de bicicleta para ir para a escola e voltar para casa?" e "durante os últimos sete dias, quanto tempo, em média, você gastou para ir de casa para escola e voltar até a sua casa?". Foram classificados como "fisicamente inativos" aqueles que relataram que em nenhum dos dias da última semana realizaram deslocamentos a pé ou de bicicleta para a escola e/ou aqueles que, independentemente da frequência semanal de prática desse tipo de atividade, relataram que a duração do deslocamento para a escola era inferior a 20 minutos (trecho de ida e volta).

As variáveis independentes foram: sexo (rapazes, moças), faixa etária (14-16; 17-19 anos), local de residência (urbana, rural), cor da pele (branca, não branca), situação ocupacional (não trabalha, trabalha), escolaridade materna ( $\leq$ 8 anos, 9-11 anos, 12 anos ou mais de estudo), porte da escola (pequeno, médio, grande), turno (diurno, noturno), série (1aa, 2ạa, 3ạ) e excesso de peso (sim, não).

O excesso de peso (sobrepeso e obesidade) foi determinado de acordo com os pontos de corte para o IMC, propostos pelo International Obesity Task Force e publicados por Cole et al. 23. A opção por esta referência de avaliação foi decorrente do seu uso mais frequente em estudos congêneres, permitindo, desse modo, maior comparabilidade dos achados deste estudo com o que está relatado na literatura especializada.

O procedimento de tabulação foi efetuado em um arquivo de dados do programa EpiData, versão 3.1 (Epidata Assoc. Odense, Dinamarca), utilizando procedimentos eletrônicos de controle de entrada de dados. Recorreu-se à dupla digitação a fim de conferir a consistência na entrada dos dados. Todos os erros de digitação identificados foram corrigidos.

Para realização das análises, utilizou-se o pacote estatístico Stata, versão 10 (Stata Corp., 
College Station, Estados Unidos), empregandose procedimentos de estatística descritiva (distribuição de frequência, média e desvio-padrão) e inferencial.

Na análise bivariada, recorreu-se à aplicação dos testes de qui-quadrado e qui-quadrado para tendência. Esse procedimento foi empregado para apresentar ao leitor uma comparação das prevalências de inatividade física nos deslocamentos entre as categorias das variáveis independentes.

$\mathrm{Na}$ análise multivariada, utilizou-se a regressão logística binária, adotando a inatividade física nos deslocamentos para escola como desfecho. Um modelo hierárquico foi empregado para estabelecer a ordem de entrada das variáveis independentes, conforme sugerido na literatura 24 . O modelo conceitual de análise adotado considerou três níveis de determinação causal (proximal, intermediário, distal). No primeiro nível, foram incluídos os fatores demográficos (faixa etária, local de residência); no segundo, foram adicionados os fatores socioeconômicos (cor da pele, ocupação, escolaridade materna) e aqueles relacionados à escola (série, turno, porte da escola); no terceiro, foi inserido o excesso de peso (Figura 1).

Figura 1

Modelo hierárquico de análise de fatores associados à inatividade física nos deslocamentos para escola.

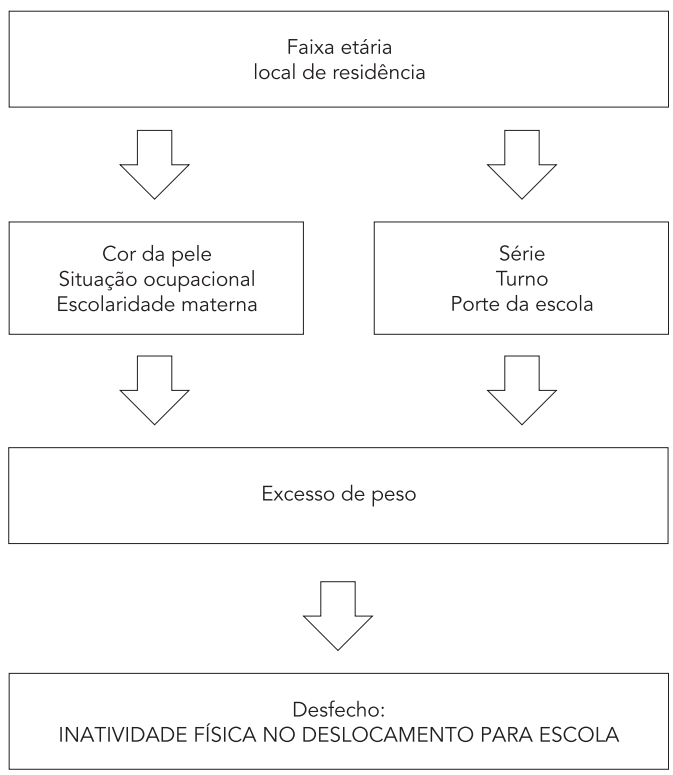

Cad. Saúde Pública, Rio de Janeiro, 26(7):1419-1430, jul, 2010
Nas análises de regressão, apenas as variáveis que tiveram valor de $\mathrm{p}$ inferior a 0,2 foram incluídas no nível subsequente de ajustamento. No modelo final de regressão, foram considerados fatores significativamente associados à inatividade física nos deslocamentos, somente aqueles para os quais o valor de $\mathrm{p}$ foi inferior a 0,05.

\section{Resultados}

Foram visitadas 76 escolas $(11 \%$ do total de escolas estaduais do estado) em 44 municípios, o que representa $23 \%$ do total de municípios pernambucanos. Do total de alunos que estavam em sala de aula no dia da coleta, 83 se recusaram a participar do estudo (1,9\%) e outros 113 responderam ao questionário, mas não aceitaram aferir medidas antropométricas. Foram efetivamente entrevistados e avaliados 4.207 estudantes com idade entre 14 e 19 anos (média de 16,8 anos; s = 1,4 ), dos quais $59,8 \%$ eram do sexo feminino. Não foram observadas diferenças estatisticamente significativas entre os participantes e aqueles que se recusaram a participar das medidas antropométricas quanto à distribuição dos sujeitos em relação ao sexo $(p=0,71)$, faixa etária $(p=0,85)$, local de residência $(\mathrm{p}=0,87)$, cor da pele $(\mathrm{p}=$ $0,32)$, situação ocupacional $(p=0,94)$ e escolaridade materna $(\mathrm{p}=0,17)$.

A Tabela 1 apresenta as características demográficas, socioeconômicas, relacionadas à escola e excesso de peso, estratificadas por sexo.

A prevalência de inatividade física nos deslocamentos para escola foi de $43 \%$ (IC95\%: 41,5$44,5)$, sendo estatisticamente $(\mathrm{p}=0,03)$ superior nos rapazes (45\%; IC95\%: 42,6-47,4), em comparação com as moças (41,6\%; IC95\%: 39,6-43,5). A proporção de adolescentes fisicamente inativos nos deslocamentos foi significativamente maior entre os adolescentes residentes na zona rural $(66,8 \%)$ e entre aqueles que relataram ter pele branca (45,7\%), em comparação, respectivamente, àqueles que residem na área urbana $(36,6 \%) \mathrm{e}$ referiram ter pele não branca $(42,1 \%)$. Adicionalmente, verificou-se que a prevalência de inatividade física no domínio dos deslocamentos está diretamente associada à escolaridade materna, reduzindo de $51,4 \%$ para $42,2 \%$, respectivamente, entre os adolescentes que relataram maior e menor escolaridade materna.

Além disso, observou-se que a frequência de inatividade nos deslocamentos foi superior entre os alunos que estudavam em escolas de pequeno porte $(57,2 \%)$, quando comparados aos que estudavam em escolas de médio (41,3\%) e grande porte $(41,7 \%)$. Similarmente, a prevalência de inatividade física foi significativamente maior 
Características demográficas, socioeconômicas, relacionadas à escola e excesso de peso dos adolescentes $(n=4.207)$

estudantes do Ensino Médio da rede pública estadual de ensino do Estado de Pernambuco, Brasil, 2006.

\begin{tabular}{|c|c|c|c|c|}
\hline \multirow[t]{2}{*}{ Variável } & \multicolumn{2}{|c|}{ Rapazes } & \multicolumn{2}{|c|}{ Moças } \\
\hline & $\%$ & $\mathrm{n}$ & $\%$ & $\mathrm{n}$ \\
\hline \multicolumn{5}{|l|}{ Faixa etária (anos) } \\
\hline $14-16$ & 35,4 & 599 & 46,4 & 1.165 \\
\hline $17-19$ & 64,6 & 1.089 & 53,6 & 1.346 \\
\hline \multicolumn{5}{|l|}{ Local de residência } \\
\hline Urbana & 78,1 & 1.311 & 79,5 & 1.983 \\
\hline Rural & 21,9 & 367 & 20,4 & 510 \\
\hline \multicolumn{5}{|l|}{ Cor da pele } \\
\hline Branca & 24,8 & 417 & 25,5 & 639 \\
\hline Não branca & 75,2 & 1.262 & 74,5 & 1.866 \\
\hline \multicolumn{5}{|l|}{ Situação ocupacional } \\
\hline Não trabalha & 69,2 & 1.157 & 84,8 & 2.119 \\
\hline Trabalha & 30,8 & 514 & 15,2 & 381 \\
\hline \multicolumn{5}{|c|}{ Escolaridade materna (anos de estudo) } \\
\hline$\leq 8$ & 69,4 & 1.086 & 74,5 & 1.771 \\
\hline $9-11$ & 22,5 & 352 & 20,2 & 480 \\
\hline$\geq 12$ & 8,1 & 127 & 5,30 & 126 \\
\hline \multicolumn{5}{|c|}{ Porte da escola (número de estudantes) } \\
\hline$<200$ & 9,0 & 152 & 8,80 & 221 \\
\hline $200-499$ & 27,0 & 456 & 25,0 & 628 \\
\hline$\geq 500$ & 64,0 & 1.079 & 66,2 & 1.662 \\
\hline \multicolumn{5}{|l|}{ Série } \\
\hline 1으 ano & 46,1 & 778 & 43,8 & 1.099 \\
\hline 2o ano & 31,0 & 522 & 32,6 & 818 \\
\hline 3o ano & 22,9 & 386 & 23,6 & 593 \\
\hline \multicolumn{5}{|l|}{ Turno } \\
\hline Diurno (manhã/tarde) & 53,9 & 908 & 60,0 & 1.506 \\
\hline Noturno & 46,1 & 778 & 40,0 & 1.002 \\
\hline \multicolumn{5}{|l|}{ Excesso de peso } \\
\hline Não & 91,1 & 1.497 & 89,6 & 2.188 \\
\hline Sim & 8,9 & 147 & 10,4 & 253 \\
\hline
\end{tabular}

entre os estudantes do primeiro ano do ensino médio (45,6\%) em comparação com aqueles matriculados no segundo $(40,1 \%)$ e terceiro ano (42\%).

A prevalência de inatividade física nos deslocamentos segundo fatores demográficos, socioeconômicos, relacionados à escola e excesso de peso está apresentada na Tabela 2, recorrendo-se à estratificação das análises por sexo.

Análises de regressão multivariáveis evidenciaram, tanto entre os rapazes quanto entre as moças, que o local de residência e a escolaridade materna são fatores significativamente associados à inatividade física nos deslocamentos (Ta- belas 3 e 4). Estudantes residentes na zona rural apresentaram maior chance de inatividade física nos deslocamentos para escola em comparação àqueles residentes em área urbana. Em relação à escolaridade materna, observou-se tendência de elevação na prevalência de inatividade física nos deslocamentos com aumento da escolaridade materna.

Identificou-se, ainda, que a faixa etária e a cor da pele entre os rapazes são variáveis significativamente associadas à inatividade física nos deslocamentos. Entre os rapazes, os estudantes com 17-19 anos e aqueles de cor de pele não branca têm menor chance de inatividade física nos des- 
Tabela 2

Proporção de adolescentes fisicamente inativos nos deslocamentos para escola de acordo com variáveis demográficas, socioeconômicas, relacionadas à escola e excesso de peso, estratificada por sexo. Pernambuco, Brasil, 2006.

\begin{tabular}{|c|c|c|c|c|}
\hline \multirow[t]{2}{*}{ Variável } & \multicolumn{2}{|c|}{ Rapazes } & \multicolumn{2}{|c|}{ Moças } \\
\hline & $\%$ & $\mathrm{n}$ & $\%$ & $n$ \\
\hline \multicolumn{5}{|l|}{ Faixa etária (anos) } \\
\hline $14-16$ & 48,6 & 289 & 41,7 & 482 \\
\hline $17-19$ & 43,1 & 468 & 41,5 & 555 \\
\hline Valor de $p^{*}$ & \multicolumn{2}{|c|}{0,031} & \multicolumn{2}{|c|}{0,905} \\
\hline \multicolumn{5}{|c|}{ Local de residência } \\
\hline Urbana & 38,5 & 503 & 35,4 & 696 \\
\hline Rural & 67,8 & 249 & 66,1 & 335 \\
\hline Valor de $p^{*}$ & \multicolumn{2}{|c|}{0,000} & \multicolumn{2}{|c|}{0,000} \\
\hline \multicolumn{5}{|l|}{ Cor da pele } \\
\hline Branca & 49,6 & 206 & 43,2 & 274 \\
\hline Não branca & 43,5 & 547 & 41,1 & 761 \\
\hline Valor de $p^{*}$ & \multicolumn{2}{|c|}{0,028} & \multicolumn{2}{|c|}{0,343} \\
\hline \multicolumn{5}{|c|}{ Situação ocupacional } \\
\hline Não trabalha & 44,5 & 513 & 42,2 & 889 \\
\hline Trabalha & 46,0 & 236 & 37,6 & 142 \\
\hline Valor de $p$ * & \multicolumn{2}{|c|}{0,567} & \multicolumn{2}{|c|}{0,091} \\
\hline \multicolumn{5}{|c|}{ Escolaridade materna (anos de estudo) } \\
\hline$\leq 8$ & 44,0 & 476 & 41,1 & 722 \\
\hline $9-11$ & 45,0 & 158 & 42,0 & 200 \\
\hline$\geq 12$ & 52,0 & 66 & 50,8 & 64 \\
\hline Valor de $p^{*}$ & \multicolumn{2}{|c|}{0,231} & \multicolumn{2}{|c|}{0,104} \\
\hline \multicolumn{5}{|l|}{ Série } \\
\hline 1 으 ano & 48,8 & 379 & 43,1 & 470 \\
\hline 2 으 ano & 41,6 & 216 & 39,2 & 320 \\
\hline 으 ano & 42,0 & 162 & 42,0 & 247 \\
\hline Valor de $p^{* \star}$ & \multicolumn{2}{|c|}{0,015} & \multicolumn{2}{|c|}{0,230} \\
\hline \multicolumn{5}{|l|}{ Turno } \\
\hline Diurno & 45,1 & 409 & 43,5 & 652 \\
\hline Noturno & 44,8 & 347 & 38,7 & 383 \\
\hline Valor de $p^{\star *}$ & \multicolumn{2}{|c|}{0,898} & \multicolumn{2}{|c|}{0,018} \\
\hline \multicolumn{5}{|c|}{ Porte da escola (número de estudantes) } \\
\hline$<200$ & 63,2 & 96 & 52,5 & 116 \\
\hline $200-499$ & 42,0 & 191 & 40,8 & 254 \\
\hline$\geq 500$ & 43,8 & 470 & 40,4 & 667 \\
\hline Valor de $p^{\star \star}$ & \multicolumn{2}{|c|}{0,000} & & \\
\hline Excesso de peso & & & & \\
\hline Não & 45,1 & 647 & 41,9 & 874 \\
\hline Sim & 43,1 & 85 & 40,0 & 134 \\
\hline Valor de $p$ * & & & & \\
\hline
\end{tabular}

* Teste do qui-quadrado para heterogeneidade;

** Teste do qui-quadrado para tendência. 
locamentos em comparação, respectivamente, aos mais jovens (14-16 anos) e estudantes que referiram cor de pele branca (Tabela 3 ).

\section{Discussão}

O presente estudo evidenciou que a prevalência de inatividade física nos deslocamentos para a escola entre os adolescentes foi relativamente alta em comparação a outros levantamentos congêneres. Em ambos os sexos, identificou-se que o local de residência e a escolaridade materna foram estatisticamente associados ao desfecho.
Além disso, a inatividade física nos deslocamentos foi significativamente associada à faixa etária e à cor da pele, entre os rapazes.

No entanto, algumas limitações precisam ser consideradas neste estudo. Não foi possível controlar algumas variáveis de confundimento relacionadas aos fatores pessoais (possuir carteira de habilitação, possuir veículo motorizado, número de veículos motorizados por domicílio) e ambientais (características das ruas, congestionamentos, barulho no tráfego, segurança no trânsito, segurança contra crimes), que podem influenciar a prática de atividade física nos deslocamentos. Outro fator que não foi mensurado,

Tabela 3

Análise de regressão logística para identificação de fatores associados à inatividade física nos deslocamentos para escola em adolescentes do sexo masculino ( $n=1.681)$. Pernambuco, Brasil, 2006.

\begin{tabular}{|c|c|c|c|c|}
\hline Variável/Categorias & $\begin{array}{l}\text { OR bruta } \\
\text { (IC95\%) }\end{array}$ & Valor de $p$ & $\begin{array}{l}\text { OR ajustada } \\
\text { (IC95\%) * }\end{array}$ & Valor de $p$ \\
\hline \multicolumn{5}{|l|}{ Faixa etária (anos) } \\
\hline $14-16$ & 1,00 & & 1,00 & \\
\hline $17-19$ & $0,80(0,66-0,98)$ & 0,031 & $0,78(0,63-0,95)$ & 0,016 \\
\hline \multicolumn{5}{|l|}{ Local de residência } \\
\hline Urbana & 1,00 & & 1,00 & \\
\hline Rural & $3,36(2,63-4,30)$ & 0,000 & $3,40(2,66-4,35)$ & 0,000 \\
\hline \multicolumn{5}{|l|}{ Cor da pele } \\
\hline Branca & 1,00 & & 1,00 & \\
\hline Não branca & $0,78(0,62-0,97)$ & 0,029 & $0,78(0,61-0,99)$ & 0,043 \\
\hline \multicolumn{5}{|l|}{ Situação ocupacional } \\
\hline Não trabalha & 1,00 & & Excluído & \\
\hline Trabalha & $1,06(0,86-1,31)$ & 0,577 & & \\
\hline \multicolumn{5}{|c|}{ Escolaridade materna (anos de estudo) } \\
\hline$\leq 8$ & 1,00 & & 1,00 & \\
\hline $9-11$ & $1,04(0,82-1,33)$ & & $1,31(1,02-1,70)$ & \\
\hline$\geq 12$ & $1,38(0,95-1,99)$ & 0,140 & $1,53(1,04-2,25)$ & 0,006 \\
\hline \multicolumn{5}{|l|}{ Série } \\
\hline 1으 ano & 1,00 & & 1,00 & \\
\hline 2으 ano & $0,74(0,60-0,93)$ & & $0,94(0,73-1,22)$ & \\
\hline 3o ano & $0,76(0,59-0,97)$ & 0,010 & $0,96(0,72-1,28)$ & 0,797 \\
\hline \multicolumn{5}{|l|}{ Turno } \\
\hline Diurno & 1,00 & & Excluído & \\
\hline Noturno & $0,99(0,81-1,20)$ & 0,898 & & \\
\hline \multicolumn{5}{|c|}{ Porte da escola (número de estudantes) } \\
\hline$<200$ & 1,00 & & 1,00 & \\
\hline $200-499$ & $0,42(0,29-0,62)$ & & $0,50(0,33-0,75)$ & \\
\hline$\geq 500$ & $0,45(0,32-0,64)$ & 0,002 & $0,56(0,39-0,83)$ & 0,076 \\
\hline \multicolumn{5}{|l|}{ Excesso de peso } \\
\hline Não & 1,00 & & Excluído & \\
\hline Sim & $0,92(0,68-1,25)$ & 0,602 & & \\
\hline
\end{tabular}

* Análise multivariável por regressão logística binária, seguindo modelo hierarquizado em três níveis. 


\begin{tabular}{|c|c|c|c|c|}
\hline Variável/Categorias & $\begin{array}{l}\text { OR bruta } \\
\text { (IC95\%) }\end{array}$ & Valor de $\mathrm{p}$ & $\begin{array}{l}\text { OR ajustada } \\
\text { (IC95\%) * }\end{array}$ & Valor de $\mathrm{p}$ \\
\hline \multicolumn{5}{|l|}{ Faixa etária (anos) } \\
\hline $14-16$ & 1,00 & & Excluído & \\
\hline $17-19$ & $0,99(0,84-1,16)$ & 0,905 & & \\
\hline \multicolumn{5}{|l|}{ Local de residência } \\
\hline Urbana & 1,00 & & 1,00 & \\
\hline Rural & $3,56(2,90-4,38)$ & 0,000 & $3,56(2,90-4,38)$ & 0,000 \\
\hline \multicolumn{5}{|l|}{ Cor da pele } \\
\hline Branca & 1,00 & & Excluído & \\
\hline Não branca & $0,92(0,76-1,10)$ & 0,341 & & \\
\hline \multicolumn{5}{|l|}{ Situação ocupacional } \\
\hline Não trabalha & 1,00 & & 1,00 & \\
\hline Trabalha & $0,82(0,66-1,03)$ & 0,091 & $0,83(0,65-1,06)$ & 0,125 \\
\hline \multicolumn{5}{|c|}{ Escolaridade materna (anos de estudo) } \\
\hline$\leq 8$ & 1,00 & & 1,00 & \\
\hline $9-11$ & $1,04(0,85-1,28)$ & & $1,30(1,05-1,62)$ & \\
\hline$\geq 12$ & $1,48(1,03-2,13)$ & 0,080 & $1,75(1,21-2,54)$ & 0,000 \\
\hline \multicolumn{5}{|l|}{ Série } \\
\hline 10 ano & 1,00 & & Excluído & \\
\hline 2 o ano & $0,85(0,71-1,02)$ & & & \\
\hline 3ㅇ ano & $0,95(0,78-1,17)$ & 0,435 & & \\
\hline \multicolumn{5}{|l|}{ Turno } \\
\hline Diurno & 1,00 & & 1,00 & \\
\hline Noturno & $0,82(0,70-0,97)$ & 0,018 & $0,87(0,73-1,05)$ & 0,148 \\
\hline \multicolumn{5}{|c|}{ Porte da escola (número de estudantes) } \\
\hline$<200$ & 1,00 & & 1,00 & \\
\hline $200-499$ & $0,62(0,46-0,85)$ & & $0,71(0,51-0,99)$ & \\
\hline$\geq 500$ & $0,61(0,46-0,81)$ & 0,007 & $0,80(0,59-1,09)$ & 0,632 \\
\hline \multicolumn{5}{|l|}{ Excesso de peso } \\
\hline Não & 1,00 & & Excluído & \\
\hline Sim & $0,92(0,73-1,17)$ & 0,517 & & \\
\hline
\end{tabular}

* Análise multivariável por regressão logística binária, seguindo modelo hierarquizado em três níveis.

mas que interfere na prática de atividade física neste domínio, é a distância de casa para escola. A distância para escola tem sido identificada como uma barreira para o deslocamento ativo e é um forte preditor do modo de transporte para escola, com longas distâncias associadas à baixa prevalência de deslocamento ativo 25,26. Diversos estudos demonstram que esses fatores estão associados à ocorrência desse desfecho 10,25,26. Outra limitação refere-se à obtenção das informações fornecidas pelos próprios adolescentes, o que pode subestimar ou superestimar o tempo de deslocamento para escola.
Deve-se salientar, também, que os dados foram coletados em uma região específica do Brasil, e os estudantes matriculados em escolas da rede pública estadual de Ensino Médio não representam a população adolescente como um todo. Assim, a generalização dos achados deste estudo pode ser limitada.

Apesar das limitações, esta investigação apresenta pontos positivos que merecem ser destacados, como a abrangência da pesquisa e os cuidados adotados no dimensionamento e seleção da amostra. Outro ponto positivo a ser levantado refere-se ao questionário utilizado na coleta dos dados, o qual foi previamente testado e apresen- 
tou boa consistência de medidas em réplicas de aplicação, além de elevada taxa de resposta.

Os resultados deste levantamento indicam que a prevalência de inatividade física nos deslocamentos para escola foi alta em comparação ao observado em estudos realizados com adolescentes dinamarqueses 13 e filipinos 27 , mas foi inferior ao verificado em levantamentos realizados com canadenses 28 e norte-americanos 29,30.

Comparando os achados para este grupo populacional com os de outros levantamentos conduzidos no Brasil, verificou-se que a prevalência de inatividade física nos deslocamentos para escola foi superior à identificada em adolescentes do Estado de Santa Catarina 18, estudantes de Pelotas (Rio Grande do Sul) 19,31 e de João Pessoa (Paraíba) 32.

No presente estudo, observou-se que a proporção de inatividade física nos deslocamentos foi significativamente maior nos rapazes em comparação com as moças. Achados semelhantes foram observados entre os adolescentes asiáticos 27, americanos 33 e espanhóis 34 . Também foi similar ao verificado nos escolares da cidade de João Pessoa ${ }^{32}$. Em contrapartida, estes resultados divergem dos apresentados por Davison et al. 26 e Bastos et al. ${ }^{31}$, que verificaram os rapazes como sendo mais propensos ao deslocamento ativo em comparação às moças.

De acordo com McDonald 35, o sexo tem pouco efeito sobre o modo de escolha dos deslocamentos para escola. Apesar de as evidências mostrarem que as meninas são, culturalmente, mais protegidas que os meninos, diversos fatores podem interferir na realização de atividades físicas nesse domínio de vida, como as escolhas do tipo de deslocamento, a distância da residência para escola, as opções de transporte e a idade.

Os resultados apresentados na presente pesquisa evidenciaram que os rapazes mais jovens tinham maior chance de serem fisicamente inativos nos deslocamentos para escola do que os mais velhos. Embora estudos mostrem que adolescentes mais velhos são mais propensos a realizarem deslocamento ativo em relação aos mais jovens 10,36, outra investigação indica o contrário 29.

Evidências disponíveis quanto à associação entre prática de atividades físicas nos deslocamentos e idade são controversas. Pesquisas apontam que os resultados têm sido inconsistentes, indicando não haver uma tendência linear 26,28. Parece que os estudantes mais jovens têm menor liberdade para ir a pé ou de bicicleta para escola em comparação aos estudantes mais velhos. Este fato pode estar associado à mobilidade dependente dos pais, parentes e amigos mais velhos, os quais utilizam meios de transporte motorizado.
Neste estudo, a prevalência de inatividade física nos deslocamentos para escola foi estatisticamente maior entre os adolescentes residentes na zona rural, quando comparados aos residentes na zona urbana. A elevada proporção de estudantes fisicamente inativos na área rural pode ser explicada pelo fato de que o acesso à escola, em muitos municípios do interior do estado, é mais limitado, em virtude da distância da residência até a escola. Além disso, essa área possui poucas escolas, tendo os alunos que se deslocarem com transportes motorizados para área urbana. De acordo com Sirard et al. 33 , escolas situadas em grandes cidades podem apresentar alta proporção de indivíduos fisicamente ativos nesse domínio porque a densidade populacional nas áreas próximas às escolas tende a ser maior.

Os resultados desta pesquisa evidenciaram, ainda, uma tendência de elevação na proporção de inatividade física nos deslocamentos conforme aumento da escolaridade materna, para ambos os sexos. Evidências disponíveis indicam que adolescentes de baixo nível socioeconômico realizam com maior frequência o deslocamento ativo, em comparação com seus pares de alto nível socioeconômico ${ }^{31}$. No entanto, os resultados diferem, dependendo do fator socioeconômico estudado ${ }^{34}$. Um levantamento conduzido por Chilló et al. 34, analisou associação de diferentes fatores socioeconômicos com a prática de atividade física nos deslocamentos em adolescentes espanhóis. Os autores verificaram que escolaridade e nível profissional materno foram os principais fatores associados à inatividade física nos deslocamentos.

Quanto às questões ligadas à cor da pele e ocorrência de inatividade física nos deslocamentos, os achados deste estudo concordam com os levantamentos apresentados na literatura. No trabalho conduzido por McDonald 36, com estudantes de 5 a 18 anos de idade, identificou-se que a inatividade física nos deslocamentos para escola foi estatisticamente associada à etnia, de modo que os indivíduos latinos e negros usavam mais o modo de deslocamento ativo para escola em comparação com os estudantes brancos. Foi observado resultado semelhante na pesquisa conduzida por Evenson et al. 29, indicando que a inatividade física nos deslocamentos foi mais prevalente entre estudantes que referiram ter cor de pele branca em comparação aos de pele não branca.

Faulkner et al. 12 sugerem que há poucas evidências relacionadas à associação da prática de atividade física no domínio dos deslocamentos e massa corporal saudável em crianças. Os autores também observaram que os estudos que incluíam medidas de massa corporal não foram signi- 
ficativamente associados à prática de atividade física nos deslocamentos, conforme observado no presente estudo.

Os achados desta investigação adicionam ao corpo de conhecimento disponível importantes evidências sobre a prevalência e os fatores associados à inatividade física nos deslocamentos para escola. Essas informações podem orientar o planejamento e a avaliação de programas educativos, semelhantes ao Safe Routes to School e ao Kids Walk-to-school, que favorecem a utilização de meios de transporte fisicamente ativo para ir à escola e voltar para casa. Particularmente para os adolescentes que residem na área rural, é necessário o desenvolvimento de ações que estimulem o deslocamento ativo para escola, como a criação de trilhas ecológicas para caminhada e rotas seguras para deslocamento usando bicicletas.

Sugere-se a realização de estudos nacionais com diferentes grupos populacionais, assim como o controle de possíveis fatores ambientais para inatividade física no domínio dos deslocamentos.

\section{Resumo}

O objetivo deste estudo foi verificar a prevalência e identificar fatores associados à inatividade física nos deslocamentos para escola em adolescentes. Trata-se de um estudo epidemiológico transversal baseado na análise secundária de dados de uma amostra de 4.207 adolescentes (14-19 anos). Os dados foram coletados por meio de um questionário previamente validado (GSHS-OMS). Foram classificados como "inativos nos deslocamentos" aqueles que relataram que não se deslocavam ativamente para ir à escola elou aqueles que o fazem, mas despendem menos de 20 minutos no trajeto de ida e volta. Observou-se que 43\% (IC95\%: 41,5$44,5)$ dos adolescentes são fisicamente inativos nos deslocamentos. Verificou-se que o local de residência e a escolaridade materna foram estatisticamente associados ao desfecho (inatividade física nos deslocamentos para escola $)(p<0,001)$. Entre os rapazes, a inatividade física nos deslocamentos foi significativamente associada à faixa etária $(p=0,02)$ e à cor da pele $(p=$ 0,04 ). A inatividade nos deslocamentos é relativamente alta em comparação a outros estudos congêneres.

Atividade Motora; Caminhada; Ciclismo; Adolescente

\section{Colaboradores}

C. M. Santos participou da concepção do estudo, redação do manuscrito e análise estatística. R. S. Wanderley Júnior efetuou a revisão de literatura, a tabulação dos dados e revisou criticamente o manuscrito. S. S. H. Barros revisou criticamente o manuscrito. J. C. Farias Júnior revisou criticamente o manuscrito, cooperando também com as análises estatísticas. M. V. G. Barros liderou a concepção do estudo, a redação do manuscrito e as análises estatísticas.

\section{Agradecimentos}

À Secretaria de Educação e Cultura de Pernambuco e às Gerências Regionais de Educação, pelo apoio logístico. Aos diretores e professores das escolas, que contribuíram para a execução da coleta dos dados. Aos integrantes do Grupo de Pesquisa em Estilos de Vida e Saúde da Escola Superior de Educação Física da Universidade de Pernambuco, que colaboraram na coleta $\mathrm{e}$ tabulação dos dados. Ao Conselho Nacional de Desenvolvimento Científico e Tecnológico (processo CNPq 486023/2006-0). 


\section{Referências}

1. Sofi F, Capalbo A, Cesari F, Abbate R, Gensini GF. Physical activity during leisure time and primary prevention of coronary heart disease: an updated meta-analysis of cohort studies. Eur J Cardiovasc Prev Rehabil 2008; 15:247-57.

2. Hu G, Qiao Q, Silventoinen K, Eriksson JG, Jousilahti P, Lindstrom J, et al. Occupational, commuting, and leisure-time physical activity in relation to risk for type 2 diabetes in middle-aged Finnish men and women. Diabetologia 2003; 46:322-9.

3. Hu G, Sarti C, Jousilahti P, Silventoinen K, Barengo NC, Tuomilehto J. Leisure time, occupational, and commuting physical activity and the risk of stroke. Stroke 2005; 36:1994-9.

4. Mai PL, Sullivan-Halley J, Ursin G, Stram DO, Deapen D, Villaluna D, et al. Physical activity and colon cancer risk among women in the California teachers study. Cancer Epidemiol Biomarkers Prev 2007; 16:517-25.

5. Conm VS, Hafdahl AR, Brown LM. Meta-analysis of quality-of-life outcomes from physical activity interventions. Nurs Res 2009; 58:175-83.

6. Adams J. Trends in physical activity and inactivity amongst US 14-18 year olds by gender, school grade and race, 1993-2003: evidence from the youth risk behavior survey. BMC Public Health 2006; 6:57.

7. Borodulin K, Laatikainen T, Juolevi A, Jousilahti P. Thirty-year trends of physical activity in relation to age, calendar time and birth cohort in Finnish adults. Eur J Public Health 2008; 18:339-44.

8. World Health Organization. Global strategy on diet, physical activity and health. http://www.who.int/ dietphysicalactivity/en/ (acessado em Ago/2009).

9. Berrigan D, Troiano RP, McNeel T, Disogra C, Bal lard-Barbash R. Active transportation increases adherence to activity recommendations. Am J Prev Med 2006; 31:210-6.

10. Timperio A, Ball K, Salmon J, Roberts R, Giles-Corti B, Simmons D, et al. Personal, family, social, and environmental correlates of active commuting to school. Am J Prev Med 2006; 30:45-51.

11. Tudor-Locke C, Ainsworth BE, Popkin BM. Active commuting to school: an overlooked source of childrens' physical activity? Sports Med 2001; 31:309-13.

12. Faulkner GE, Buliung RN, Flora PK, Fusco C. Active school transport, physical activity levels and body weight of children and youth: a systematic review. Prev Med 2009; 48:3-8.

13. Cooper AR, Wedderkopp N, Wang H, Andersen LB, Froberg K, Page AS. Active travel to school and cardiovascular fitness in Danish children and adolescents. Med Sci Sports Exerc 2006; 38:1724-31.

14. Heelan KA, Donnelly JE, Jacobsen DJ, Mayo MS, Washburn R, Greene L. Active commuting to and from school and BMI in elementary school children preliminary data. Child Care Health Dev 2005; 31:341-9.

15. Hamer M, Chida Y. Active commuting and cardiovascular risk: a meta-analytic review. Prev Med 2008; 46:9-13.
16. U.S. Department of Health and Human Services. Healthy people 2010: objectives for improving health. Washington DC: U.S. Public Health Service; 2000.

17. Santos CM, Barbosa JMV, Cheng LA, Wanderley Júnior RS, Barros MVG. Atividade física no contexto dos deslocamentos: revisão sistemática dos estudos epidemiológicos realizados no Brasil. Rev Bras Ativ Fís Saúde 2009; 14:13-20.

18. Lopes AS, Pires Neto CS. Estilo de vida de crianças com diferentes características étnico-culturais do Estado de Santa Catarina, Brasil. Rev Bras Ativ Fís Saúde 2001; 6:6-16.

19. Hallal PC, Bertoldi AD, Gonçalves H, Victora CG. Prevalência de sedentarismo e fatores associados em adolescentes de 10-12 anos de idade. Cad Saúde Pública 2006; 22:1277-87.

20. Silva KS, Lopes AS. Excesso de peso, pressão arterial e atividade física no deslocamento à escola. Arq Bras Cardiol 2008; 91:93-101.

21. Instituto Brasileiro de Geografia e Estatística. Censo demográfico. Rio de Janeiro: Instituto Brasileiro de Geografia e Estatística; 2000.

22. Lohman TG, Roche AF, Martorell R. Anthropometric standardization reference manual. Champaign: Human Kinetics Books; 1991.

23. Cole TJ, Bellizzi MC, Flegal KM, Dietz WH. Establishing a standard definition for child overweight and obesity worldwide: international survey. BMJ 2000; 6:1240-3.

24. Dumith SC. Proposta de um modelo teórico para a adoção da prática de atividade física. Rev Bras Ativ Fís Saúde 2008; 13:52-62.

25. Bringolf-Isler B, Grize L, Mäder U, Ruch N, Sennhauser FH, Braun-Fahrländer C, et al. Personal and environmental factors associated with active commuting to school in Switzerland. Prev Med 2008; 46:67-73.

26. Davison KK, Werder JL, Lawson CT. Children's active commuting to school: current knowledge and future directions. Prev Chronic Dis 2008; 3:A100.

27. Tudor-Locke C, Ainsworth BE, Adair LS, Popkin BM. Objective physical activity of Filipino youth stratified for commuting mode to school. Med Sci Sports Exerc 2003; 35:465-71.

28. Pabayo R, Gauvin L. Proportions of students who use various modes of transportation to and from school in a representative population-based sample of children and adolescents, 1999. Prev Med 2008; 46:63-6.

29. Evenson KR, Huston SL, McMillen BJ, Bors P, Ward DN. Statewide prevalence and correlates of walking and bicycle to school. Arch Pediatr Adolesc Med 2003; 157:887-92.

30. Centers for Disease Control and Prevention. Barriers to children walking to or from school - United States, 2004. MMWR Morb Mortal Wkly Rep 2005; 54:950-80.

31. Bastos JP, Araújo CLP, Hallal PC. Prevalence of insufficient physical activity and associated factors in brazilian adolescents. J Phys Act Health 2008; 5:777-94. 
32. Silva KS, Lopes AS, Silva FM. Atividade física no deslocamento à escola e no tempo livre em crianças e adolescentes da cidade de João Pessoa, PB, Brasil. Rev Bras Ciênc Mov 2007; 15:61-70.

33. Sirard JR, Ainsworth BE, McIver KL, Pate RR. Prevalence of active commuting at urban and suburban elementary schools in Columbia, SC. Am J Public Health 2005; 95:236-7.

34. Chillón P, Ortega FB, Ruiz JR, Pérez IJ, Martín-Matillas M, Valtueña J, et al. Socio-economic factors and active commuting to school in urban Spanish adolescents: the AVENA study. Eur J Public Health 2009; 19:470-6.
35. McDonald NC. Children's mode choice for the school trip: the role of distance and school location in walking to school. Transportation 2008; 35:23-35.

36. McDonald NC. Critical factors for active transportation to school among low-income and minority students: evidence from the 2001 National Household Travel Survey. Am J Prev Med 2008; 34:341-4.

Recebido em 27/Out/2009

Versão final reapresentada em 21/Abr/2010

Aprovado em 04/Mai/2010 